\title{
FORMALIZATION AND DATA ENRICHMENT FOR AUTOMATED EVALUATION OF BUILDING PATTERN PRESERVATION
}

\author{
Xiang Zhang ${ }^{\text {a, b, } * \text {, Jantien Stoter }}{ }^{\mathrm{c}, \mathrm{d}}$, Tinghua $\mathrm{Ai}^{\mathrm{b}}$, Menno-Jan Kraak ${ }^{\mathrm{a}}$ \\ a ITC, University of Twente, the Netherlands \\ \{xzhang, kraak\}@itc.nl \\ ${ }^{\mathrm{b}}$ School of Resource and Environment Sciences, Wuhan University, China \\ tinghua_ai@tom.com \\ ${ }^{\mathrm{c}}$ Delft University of Technology, the Netherlands \\ ${ }^{\mathrm{d}}$ Kadaster, Apeldoorm, the Netherlands \\ j.e.stoter@tudelft.nl
}

KEY WORDS: Automated Evaluation, Building Pattern Preservation, Pattern Matching, Constraint Formalization

\begin{abstract}
:
Automated evaluation of generalization output relies to a large extent on that requirements (e.g. specifications, constraints) being formalized in machine-readable formats. Previous studies suggest that the formalization and automated evaluation are relatively easier for legibility constraints (improve the readability of maps) than for preservation constraints (preserving important real-world phenomena). Three major difficulties, i.e., pattern classification and characterization, pattern matching, and constraint formalization, in the automated evaluation of building pattern preservation constraint are analyzed in this paper. A classification of available building patterns is reviewed based on a previous work. In addition, the transition events describing allowed changes for building patterns to preserve during generalization are obtained through the study of existing maps series (from 1:10k to 1:100k). Based on the obtained knowledge on pattern types and acceptable transition events, an approach to automatically match corresponding building patterns at different scales is presented. The methodology proposed is validated by applying it to the interactively generalized data. The result shows promising results and also further improvement in order to apply the method in an overall evaluation to indicate acceptable generalization solutions.
\end{abstract}

\section{INTRODUCTION}

In the map generalization process small scale map is generated from a large scale map. This intelligent information management process involves a combination of data reduction and simplification related techniques in order to suppress unnecessary detail. At the same time geographic patterns are emphasized to achieve a clear view of information that resembles the original data as much as possible. Because building patterns are significant for topographic maps, preservation of building patterns is an important cartographic constraint in the generalization process. It aims at keeping important real-world entities by discerning interesting patterns such as urban and rural structures.

This paper studies the automated evaluation of building pattern preservation in generalization. Automated evaluation of generalization output aims to assess (i.e. measure) whether or to what extent the output satisfies the cartographic constraints according to automatically derived indicators (e.g. size and shape). Automated evaluation relies firstly on the formalization of the specifications (i.e. constraints). From related work (Burghardt et al., 2007; Stoter, et al., 2009a) we can conclude that preservation constraints (e.g. on networks, patterns, and spatial distributions) are more difficult to formalize and to evaluate automatically than legibility constraints such as minimum dimension of an object required to distinguish it on the map. In this paper, formalization and data enrichment techniques for the automated evaluation of building pattern preservation are examined. In this evaluation, generalized building patterns are compared with the patterns in the original data to see if the generalized patterns meet the specification of building pattern preservation.

Difficulties in automated evaluation of building pattern preservation are manifold. First, the existing specifications concerning building patterns originally intended for interactive generalization are not easy to formalize since they are specifically meaningful for cartographers. A cartographic constraint says for example that building alignments should be preserved, which can be interpreted by cartographers so that they can apply generalization according to their knowledge or experience. Formal knowledge for computers to measure and characterize the patterns and to describe their change at scale transitions is required for automated evaluation but is not yet available. Therefore, existing specification need to be enriched for automated processes.

Second, building patterns are not stored as database objects in common topographic datasets. Consequently to automatically evaluate generalized datasets on building pattern preservation, the datasets have to be enriched with building pattern objects identified through pattern recognition techniques, or visual inspection..

A third difficulty in automating the evaluation of building pattern preservation is the lack of explicit links between correspondences at different scales. In the context of the evaluation of generalization output, the links are especially necessary for the automated evaluation of preservation

\footnotetext{
* Corresponding author. Email: xiangzhangchina@gmail.com
} 
constraints (Stoter et al., 2009a) to allow the system to know which pattern objects at different scales represent the same building group in reality.

The links can be created via generalization operators as part of the generalization process (during the process it is still known which representations are generalized into which representations at smaller scales) and via data matching (Hampe et al., 2004). The latter (data matching) is the only choice in our case as the links generated by generalization processes are not available for the datasets to be evaluated.

The following sections focus on addressing the above described difficulties.

\section{CLASSIFICATION AND CHARACTERIZATION OF BUILDING PATTERNS}

This section reviews a typology of different building patterns which is discussed in detail in our another paper (Zhang et al., 2010), where algorithms to detect them are also presented. A UML class diagram of the proposed typology is shown in Figure 1.

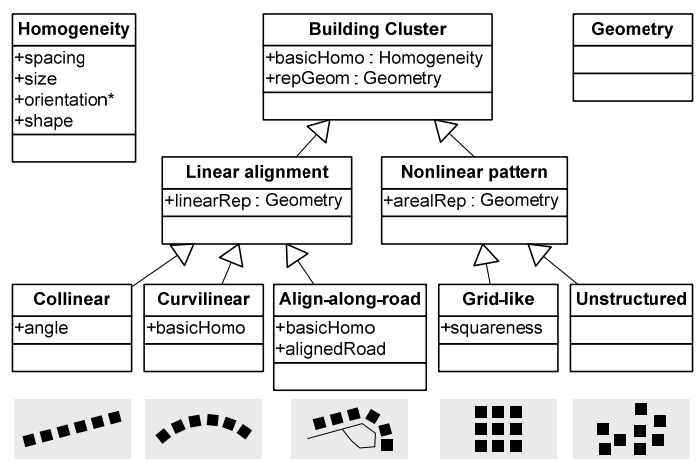

Figure 1. A UML model of the proposed pattern classification and examples of each pattern type

The UML diagram models the patterns of the typology with their representational geometries and characteristics (which can automatically be detected by the algorithms proposed in Zhang et al. (2010)). We distinguish five types of patterns, namely three linear patterns (collinear, curvilinear, and align-along-road alignments) and two nonlinear patterns (grid-like patterns and unstructured clusters). Properties all these types have in common are the representational geometries (denoted as 'repGeom') and the homogeneities (denoted as 'basicHomo') in the UML model. Two types of representational geometries are used:

- $\quad$ Linear representations (denoted as 'linearRep’): Skeletons of linear alignments; the skeletons are generated from the MST of building centers (Zhang et al., 2010);

- Areal representations (denoted as 'arealRep'): Convex hulls of nonlinear patterns; buffers of the abovementioned skeletons in some cases (see Section 3.3).

The common attribute 'basicHomo' consists of the homogeneity of spacing (between proximate buildings), size, orientation, and shape. These homogeneities can be calculated using the concept of standard deviation, reflecting the regularity of these properties (Ruas and Holzapfel, 2003). Note however that orientation should be implemented differently for curvilinear and align-along-road patterns. This is why these two classes override 'basicHomo' from their parent class. For example, the orientation is homogeneous for a collinear pattern if all buildings in the pattern have the same orientation; while it is homogeneous for a curvilinear pattern only if the orientations of buildings vary right according to the 'path' (Figure 2(2)).
Besides, specific characteristics in the model are explained: the 'angle' of a collinear alignment is the main angle of its skeleton; for each align-along-road pattern, a 'alignedRoad' should be assigned; the 'squareness' characterize the degree to which buildings align in rectangles.

It has to be noted that, these characteristics are not used to classify groups of buildings into different patterns; the pattern types are instead detected using different ad-hoc algorithms. The characteristics are calculated after the patterns have been detected, to describe the quality of the detected patterns.

\section{METHODOLOGY FOR IDENTIFYING, FORMALIZING AND EVALUATING BUILDING PATTERN PRESERVATION}

Now the building patterns are detected from data and characterized by homogeneity measures, the next step is to assess whether the generalized map preserves the homogeneity properties of the initial patterns in an acceptable way. This requires firstly knowledge on which patterns at different scales represent the same real-world entities and how these change at scale transitions (addressed in Section 3.1 to 3.3), and secondly ways to (a) automatically evaluate the constraints and (b) to interpret the quantitative evaluation results (Section 3.4).

\subsection{Identification and formalization of changes of building} patterns during generalization

A visual analysis of topographic map series was carried out for different purposes: (1) to understand why and how building patterns change at scale transitions; (2) to enrich the available written specifications for generalizing building patterns. In this analysis we identified the homogeneity classes based on pattern characteristics in the original data and try to match these with patterns in the generalized map to describe and quantify the change of patterns at scale events, i.e. do they diminish, are they preserved or are they transformed?

For this visual analysis two scale transitions (1:10k to $1: 50 \mathrm{k}$ and $1: 50 \mathrm{k}$ to $1: 100 \mathrm{k}$ ) in the topographic map series of the Netherlands have been studied. The map at scale 1:10k (supported with an object oriented database, called TOP10NL) is the most detailed topographic data, from which map at scale 1:50k (supported with TOP50NL database) is interactively generalized. The map at scale $1: 100 \mathrm{k}$ (supported with TOP100NL database) is interactively generalized from $1: 50 \mathrm{k}$ map. By comparing building patterns at these transitions, the following knowledge on their changes (as used by cartographers in the interactive generalization process) was obtained (see for examples Figure 2).

In general, we observe three forms of pattern changes at scale transitions. First, some building patterns are diminished (undetectable). Second, some patterns are transformed into built-up areas (e.g. 1A in Figure 2). The third important observation is that the group of buildings constituting a building pattern is generalized (i.e. simplified, typified, eliminated etc), resulting in a change of the pattern.

In our research on automated evaluation we specifically focus on tolerated changes to building patterns as a result of these changes, where the cases of building patterns being diminished or transformed into built-up areas are visually qualified as patterns being depressed and they are therefore out of the scope of this paper. Section 3.2 further explores how the patterns are preserved (or actually, which changes are allowed to preserve the pattern), resulting in formalized transition events. 


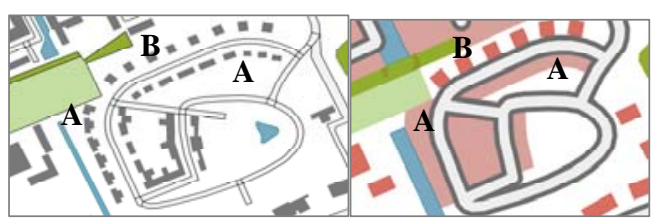

(1) From $10 \mathrm{~K}$ to $50 \mathrm{~K}$

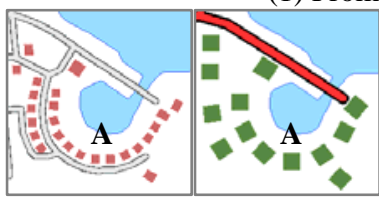

(2) From $50 \mathrm{~K}$ to $100 \mathrm{~K}$

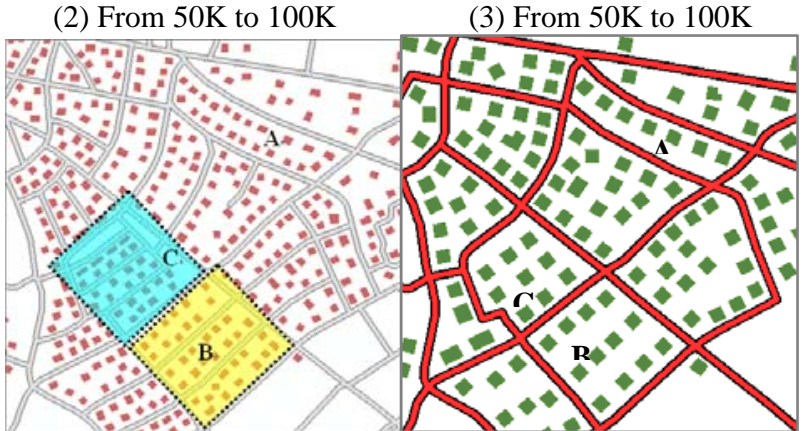

(4) From $50 \mathrm{~K}$ to $100 \mathrm{~K}$

Figure 2. The cases illustrating typical changes of building patterns at scale transitions in map series of the Netherlands' Kadaster

\subsection{Formalizing observed transition events of pattern preservation}

From closer observations of the above visual analysis of the map series, a set of transition events describing allowed changes for building pattern preservation are formalized in

Table 1. Representative cases of each observed event are exemplified in Figure 2. Note that the multiplicity of the transition events is at the pattern level rather than at the building level, indicating one- or many-to-one relationships between the pattern objects. The transition events and their possible causal factors are detailed as follows.

Event 1 shows the simplest transitions (1:1 relationship), where each ungeneralized pattern type matches with a generalized pattern of the same type, because the pattern remains significant after the generalization of buildings. For example, an align-along-road pattern can be matched to another one of the same type (1B in Figure 2). It is the same for curvilinear patterns (2A in Figure 2) and for unstructured clusters (3B in Figure 2).

Event 2 can be observed in situations where an unstructured cluster in a larger scale map is enhanced (becoming a grid pattern) during the generalization (e.g. 3A in Figure 2). Events 3-5 describe the areas where the density of buildings enclosed by roads is relatively too high. Since major roads were kept during the generalization, some of the building patterns are replaced by a row of collinear pattern due to the competition of space and legibility constraints. In events 2-4, 1:1 relationships can be found between initial and generalized patterns (i.e. a pattern object is converted as a whole into another pattern object); while event 5 (4A in Figure 2) exhibits an n:1 relationship ( $\mathrm{n} \geq 2$; several pattern objects are changed into one pattern object ). Event 4 describes that a grid pattern can become a collinear alignment but not a curvilinear pattern after the generalization.

Events 6-8 describe the cases where the change of patterns is mainly caused by the removal of streets and roads between and surrounding the patterns. This usually occurs in urban areas where the road network is highly dense and roads are selected during generalization. In such areas building patterns in nearby blocks can be aggregated or typified into bigger ones and the n:1 relationship is usually observed (e.g. 4B in Figure 2). Event 8 is shown by $4 \mathrm{C}$ in Figure 2.

Align-along-road patterns are somewhat special, as they rely on aligned roads and are sometimes similar to collinear and curvilinear patterns except their relationships to roads. Event 9 describes a situation where align-along-road patterns change with the removal of the aligned roads, which leads to the change of the patterns into collinear or curvilinear alignments.

All the formulated transition events can be observed for both transitions (i.e. $10 \mathrm{k}$ to $50 \mathrm{k}$ and $50 \mathrm{k}$ to $100 \mathrm{k}$ ). The observed events formalize the multiplicity relationships and allowed changes from one type of patterns into another to preserve important pattern characteristics. Although these transition events are empirically observed and are subject to further refinement, transitions that are considered as unacceptable (e.g. grid to unstructured) are excluded. As a result, they can be used to further enrich the available written specifications for building pattern preservation to make these suitable for automated generalization and evaluation.

In practice, the distinction between the events may not be as strict, mainly due to the uncertainty in the recognition and generalization of the patterns. A building pattern can be seen as an unstructured cluster, a grid pattern, or multiple rows of linear alignments; an align-along-road type can also be a linear alignment if the road were removed. However, this does not influence the matching because all possible transitions from one type into another are covered by the transition events while impossible ones are excluded. For example, 2A in Figure 2 can be seen as either 'curvilinear to curvilinear' (event 1 ) or 'alignalong-road to curvilinear' transition (event 9); 4B can be described either as three unstructured clusters changing into three rows of linear alignments (event 3 ) or as six rows of linear alignments changing into one grid pattern (event 6 ).

Table 1. Observed transition events of building pattern preservation at scale transitions

\begin{tabular}{|c|c|c|c|c|}
\hline \multirow[t]{2}{*}{ Event } & 1:10k & \multirow[t]{2}{*}{ Multiplicity } & $1: 50 k$ & \multirow{2}{*}{$\begin{array}{l}\text { Examples shown } \\
\text { in Figure } 2\end{array}$} \\
\hline & $1: 50 \mathrm{k}$ & & 1:100k & \\
\hline 1. & Each type of patterns & $1: 1 \rightarrow$ & The same type & 1B; 2A; 3B \\
\hline 2. & Unstructured cluster & $1: 1 \rightarrow$ & Grid pattern & $3 \mathrm{~A}$ \\
\hline 3. & Unstructured cluster & $1: 1 \rightarrow$ & Linear alignment & 4B \\
\hline 4. & Grid pattern & $1: 1 \rightarrow$ & Collinear alignment & $\mathrm{x}$ \\
\hline 5. & Linear alignments & $\mathrm{n}: 1 \rightarrow$ & Linear alignment & $4 \mathrm{~A}$ \\
\hline 6. & Linear alignments & $\mathrm{n}: 1 \rightarrow$ & Grid pattern & 4B \\
\hline 7. & Linear alignments & $\mathrm{n}: 1 \rightarrow$ & Unstructured cluster & $\mathrm{x}$ \\
\hline 8. & Nonlinear clusters & $\mathrm{n}: 1 \rightarrow$ & Nonlinear cluster & $4 \mathrm{C}$ \\
\hline 9. & Align-along-road pattern & $1: 1 \rightarrow$ & Collinear/curvilinear pattern & $2 \mathrm{~A}$ \\
\hline
\end{tabular}




\subsection{Method for automatic matching process}

The matching process consists of two sub-processes: geometric and characteristic matching, which is based on two kinds of previously enriched information, namely the pattern descriptions and the knowledge on the transition events. The pattern descriptions include pattern type information, representational geometries and homogeneity properties (Section 2). The representational geometries of the detected pattern objects are used mainly in the geometric matching process. The obtained transition events are in general used as part of the characteristic matching process: when a generalized pattern object is geometrically matched with an initial pattern object, type information concerning the two patterns is checked with respect to the transition events. If the type information is consistent with the transition events, the matching pair is selected as a candidate for further matching; if otherwise the matching of the two fails. In this latter case, one can conclude that the pattern is not preserved during generalization. It is worth noting that the buildings to be evaluated are partitioned by road networks and each step of the automatic matching is restricted to buildings within a partition cell. The technical detail of the process is described as follows.

The geometric matching deals with the similarity between geometries of the patterns to be matched. In the matching of the same type patterns (event 1 ), the similarity can be measured by distances like nearest distance, Hausdorff distance, and Fréchet distance (Alt and Godau, 1995). To match polygons (nonlinear patterns), another similarity measure (i.e. contrast model) developed by Tversky (1977) is appropriate. In the cases events 3-7 where linear alignments are matched with nonlinear clusters or several linear alignments are matched to one linear alignment, buffers of skeletons should be used instead of skeletons alone for representing linear alignments to improve the matching result. In our first experiment (Section 4), we used nearest distance and the contrast model as similarity measures to match between linear and polygonal representational geometries respectively, for simplicity reasons.

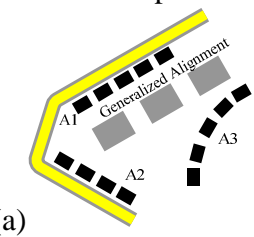

Matching between alignments (b)

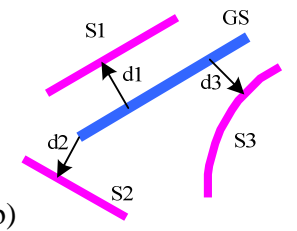

Geometric representation of the patterns
Figure 3. A scenario illustrating the problem of matching building patterns

The result from geometric matching can be refined by characteristic matching, especially when the result contains several candidates. When matching the patterns of the same type, the characteristics like pattern type, align angle, curvature and aligned road are of great importance. For example, Figure 3a describes a possible case where a generalized collinear alignment (gray buildings in Figure 3a) is (potentially) matched with two collinear patterns (A1 and A2) and a curvilinear one, as the distances (d1, d2 and d3 in Figure $3 b$ ) are similar. Such a case is possible due to the displacement of A1 caused by the widened symbol of the road. In this case, pattern type is firstly used to filter out the curvilinear pattern A3, and then align angle is calculated based on the skeletons of the alignments (angle of GS is similar to S1 than to S2), leading to a correct match between the generalized alignment and A1.

\subsection{Automated evaluation and interpretation of the evaluation results}

3.4.1 Evaluation by computing changes between matched building pattern objects

After all the required information has been collected, the automated evaluation is carried out by firstly computing characteristics defined in Section 2 for each matched building pattern objects that represent the same real-world building group, and then comparing the measured characteristics between the two pattern objects. The interpretation of the quantitative comparison is described in Section 3.4.2.

To be more concrete, the computed characteristics for both initial and generalized building patterns could be a set of separate values (e.g. homogeneity of space, size, etc.) or an aggregated value (summary of separate homogeneity values). The implementation (Section 4) is based on separate characteristics in order to demonstrate the main idea. Future work will discuss and apply the aggregation of the characteristics. Note also that if matched patterns are of the same type, then all their (common and specific) characteristics are well-matched, and then the evaluation is simply comparing their measured homogeneity values in a pair-wise manner; if they are of different types, only 'basicHomo' is compared. In the cases where $n(n \geq 2)$ initial patterns are linked to one generalized pattern, the initial homogeneities are computed by weighted average of each initial pattern; the weighting is based on lengths of skeletons for linear patterns, and on areas of convex hulls for nonlinear patterns.

3.4.2 Interpret the quantitative evaluation results The next key issue of the automated evaluation of building pattern preservation is to decide the acceptable values for the pattern characteristics after the generalization, based on which the quantitative evaluation results can be qualified into a human readable format (e.g. 'acceptable' or 'unacceptable'). According to Bard (2004) an idealized evolution function (Figure 4a) can be specified for each preservation constraint, where target characteristic values should be equal to initial values. In order to be more flexible, an acceptable range (tolerance) is introduced (dark gray areas in Figure 4).

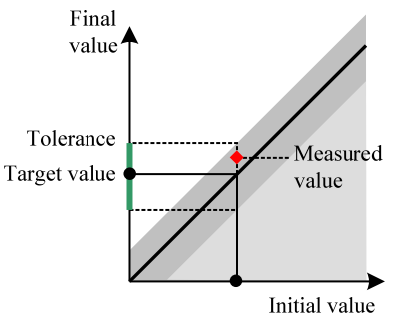

(a) Evolution function

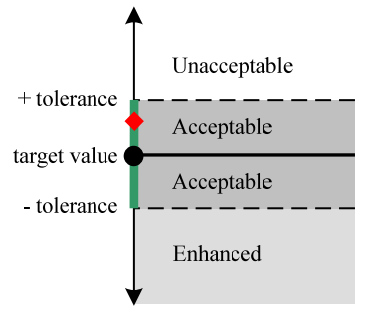

(b) Interpretation function
Figure 4. Idealized evolution function and interpretation function of building pattern preservation constraints (modified from Bard, 2004)

In our research, we slightly modify the interpretation function as proposed by Bard (2004) to be more appropriate for the pattern preservation constraint as follows. If a measured homogeneity property falls into the dark gray area in Figure 4b (|MeasuredVal - TargetVal $\mid \leq$ tolerance), then this property is considered as being well preserved (marked 
as 'acceptable'). This is motivated by the fact that small deviations are tolerated by human eyes. If the value is larger than 'target value' by a unit of tolerance, the preservation is regarded 'unacceptable'; while if the value is less than 'target value' by a unit of tolerance (light gray area in Figure 4b), the property is regarded to be 'enhanced' rather than unacceptable. This is because the building patterns can be improved by reducing the homogeneity values (i.e. improve the regularities).

\section{IMPLEMENTATION AND VALIDATION OF THE METHODOLOGY}

This section implements and validates the methodology as presented in Section 3 by applying them to a test case, to show the feasibility of the concepts. The test case consists of two datasets different from the datasets used for the visual analysis (Section 2): one is a Dutch topographic dataset at $1: 10 \mathrm{k}$ (Figure 5a); another dataset (TOP50NL) is interactively generalized from the TOP10NL (see Figure 5b). The idea is that the evaluation results from applying the methodology to interactively generalized datasets should indicate that the building patterns detected in the data are preserved sufficiently. The test results of automatic pattern matching (Section 4.1) and of validation the automated evaluation (Section 4.2) are presented.

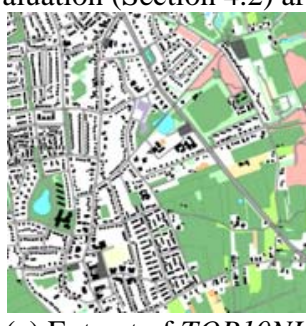

(a) Extract of TOP10NL

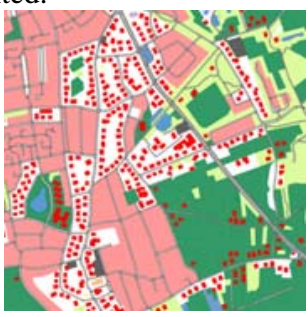

(b) Extract of TOP50NL
Figure 5. Test case for validating our evaluation methods

\subsection{Results of pattern matching}

We applied the method for automatic matching as described in Section 3.3. First, the patterns were detected using the algorithms in Zhang et al. (2010); then the data matching was carried out. In most cases, one partition cell contains only one to two detected patterns, so around $94 \%$ of the patterns (48 out of 51) detected in TOP50NL were correctly matched with their correspondences in TOP10NL. There are $53 \%$ of the patterns (68 out of 129) detected in TOP10NL mismatched. This is expected since most of the mismatch is caused by buildings transformed into built-up areas (light red areas in Figure 5b); the remaining mismatch is due to the absence of correspondences in TOP50NL. In the latter case the building patterns may have been diminished to satisfy other important constraints (e.g. minimum distances). There are also cases where some patterns (e.g. unstructured clusters) were correctly matched with others of different types (e.g. collinear alignments) and where n:1 relationships were correctly created.

\subsection{Results of characterizaion and evaluation}

The homogeneities were calculated for all matched patterns, and some rather simple examples (1:1 relationship) are illustrated (see Figure 6 and Figure 7). Some of the homogeneities were computed using coefficient of variance (std/mean) rather than standard deviation (std), as is suggested by Zhang et al. (2010). Homogeneity of orientation, however, should be computed by std, as it is a cyclic variable and is thus meaningless to calculate the coefficient of variance for it.

These figures show that the illustrated patterns are enhanced during the generalization and that at the same time the values for the homogeneity of spacing, size, and orientation are generally reduced. These results show that the proposed methods for automated evaluation of building pattern preservation are promising. In order to see if the methods can draw meaningful statement about the whole test case, regression analysis was carried out for all matched patterns. In a second step regression functions indicating the relationships between initial and target values were established concerning the homogeneity of spacing, size, and orientation (Figure 8).

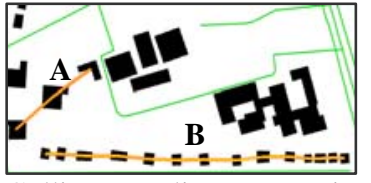

Collinear alignments in TOP10NL

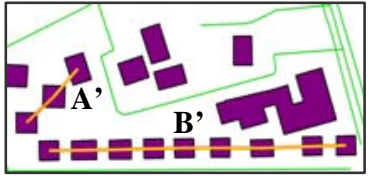

Collinear alignments in TOP50NL

\begin{tabular}{|l|l|l|l|}
\hline ID & $\begin{array}{l}\text { Spacing } \\
\text { (std/mean) }\end{array}$ & $\begin{array}{l}\text { Size } \\
\text { (std/mean) }\end{array}$ & Orientation (std) \\
\hline A & 0.06 & 0.32 & $9.74^{\circ}$ \\
\hline A $^{\prime}$ & 0.02 & 0.11 & $8.99^{\circ}$ \\
\hline B & 0.52 & 0.26 & $5.57^{\circ}$ \\
\hline B' & 0.35 & 0.09 & $0.00^{\circ}$ \\
\hline
\end{tabular}

Figure 6. Changes of the characteristics of linear patterns at a scale transition
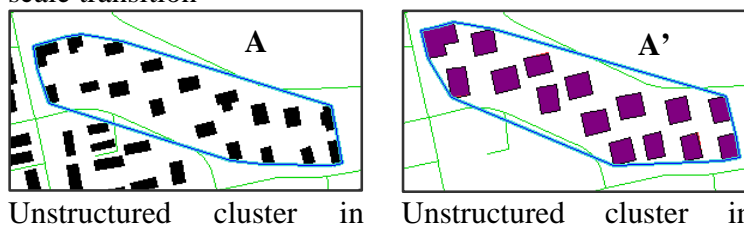

TOP10NL

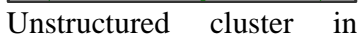
TOP50NL

\begin{tabular}{|l|l|l|l|}
\hline ID & Spacing & Size & Orientation \\
\hline A & 0.22 & 0.22 & $2.03^{\circ}$ \\
\hline A $^{\prime}$ & 0.19 & 0.19 & $1.17^{\circ}$ \\
\hline
\end{tabular}

Figure 7. Changes of the characteristics of unstructured clusters at a scale transition

These figures confirm that homogeneity values of the patterns are significantly reduced (most of the data points are below the idealized preservation function in Figure 8). This reduction means that the patterns become more homogeneous analysis of the test datasets: most of the preserved building patterns become more regular in terms of spacing, size, orientation, and shape. This is mainly due to the preservation constraint (i.e. preserve or enhance the homogeneity); legibility constraint mainly increases the minimum size and simplifies the shape of the buildings.

However, the figures also show the homogeneity values for orientation are not significantly reduced. We can see that around half of the data points in Figure 8 (leftmost) are above the idealized function, and most of the deviations are less than $5^{\circ}$. This fact can be explained because deviations ranging from $0^{\circ}$ to $10^{\circ}$ are less detectable for human eyes, and hence it is acceptable to introduce this noise during the interactive generalization. A second reason why some orientation homogeneity values increase is that some patterns detected in TOP50NL add new buildings as their elements, and these newly added buildings contribute a lot to the rise of orientation std values. In addition, due to the minimum 
distance constraint in some cases, some buildings in a pattern rotate themselves, and this also increases the orientation homogeneity. In order to formalize allowed deviations for the homogeneity of orientation, a tolerance should be derived from the training data using statistical analysis.

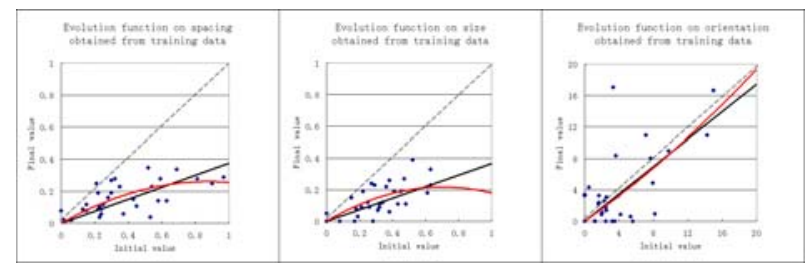

Figure 8. Evolution functions obtained from the test datasets; black line - linear function; red curve - nonlinear function; dotted line - idealized preservation function

\section{DISCUSSION AND CONCLUSIONS}

This paper studies supported methods and techniques for automated evaluation of the building pattern preservation constraint. The automated evaluation of this constraint evaluation is currently impeded by issues concerning pattern detection, pattern matching, and constraint formalization. We address these issues by firstly summarizing previously obtained results in pattern detection and characterization (Zhang et al., 2010), on which the pattern matching and evaluation are based. The classification and characterization of building patterns formalizes our knowledge on building patterns. The proposed methodology also contributes to the automated evaluation of preservation constraints in general. Then we find that the matching of building patterns is not straightforward in the sense that a type of pattern may become another type. As a result, acceptable changes (transition events) for building patterns are formulated by studying existing map series (see Table 1). This is the second valuable finding of this work, and thereby we suggest that these events could enrich the written specifications for automated processes. Later, a two-step matching process is proposed based on the enriched pattern descriptions and the transition events.

The presented methodology are implemented and validated by applying them to interactively generalized data. The major conclusion is that the methodology is promising in automated evaluation of building pattern preservation as confirmed by the validation results. The results confirm again that the pattern detection and characterization methods in Zhang et al. (2010) are consistent with our perception; the detection method is applicable to non-Dutch maps as various patterns were successfully detected in maps of France, Spain, and China. The results also indicate that the automatic matching is in itself sufficient to automatically evaluate the constraint in the case of patterns being preserved; it suggests also that a more generic framework and automatic measures would be appreciated to cover the automated evaluation of building patterns being diminished or transformed to built-up areas, although the pattern preservation constraint can be identified visually as being violated for these two cases.

For the interpretation method, the evaluation functions and tolerances in our current approach have to be decided by users and the function forms are still oversimplified as reveal by the test results. A solution to this deficiency is to determine a more adaptive evolution functions from training data. Therefore further research will employ this solution to optimize the evolution functions in order to better interpret the evaluation results.
Future work can integrate the evaluation approach with the evaluation of other constraints to obtain overall values, indicating acceptable generalization solutions. In addition, the current formalized transition events still have some overlaps and could be further optimized.

\section{ACKNOWLEDGMENTS}

The work is supported by the National High-Tech Research and Development Plan of China under the grant No. 2007AA12Z209, and the National Science Foundation, China under the grant No. 40971242. International Institute for GeoInformation Science and Earth Observation (ITC), University of twente, which funds the first author as a $\mathrm{PhD}$ student is gratefully acknowledged.

\section{REFERENCES}

Alt, H. and Godau, M., 1995. Computing the Fréchet distance between two polygonal curves. Int $J$ of Computational Geometry and Applications, 5(1-2), pp. 7591.

Bard, S. and Ruas, A., 2004. Why and How Evaluating Generalised Data? In: Developments in Spatial Data Handling (SDH'04) (Springer-Verlag), pp. 327-342.

Bobzien, M., Burghardt, D., Petzold, I., Neun, M. and Weibel, R., 2008. Multi-representation Databases with Explicitly Modeled Horizontal, Vertical, and Update Relations. Cartography and Geographic Information Science, 35(1), pp. 3-16.

Burghardt, D., Schmidt and S., Stoter, J., 2007. Investigations on cartographic constraint formalisation. In 10th ICA workshop on generalisation and multiple representation, Moscow, 2007.

Hampe, M., Anders, K. and Sester, M., 2003. MRDB applications for data revision and real-time generalization. In Proceedings of the 21st International Cartographic Conference, pp. 192-202.

Mascret, A., Devogele, T., Berre, I. L. and Hénaff, A., 2006. Coastline matching process based on the discrete Fréchet distance. In: Riedl, A.; Kainz, W. \& Elmes, G. A. (ed.), Proceedings of the 12th International Symposium on Spatial Data Handling, pp.383-400.

Ruas, A. and Holzapfel, F., 2003. Automatic characterisation of building alignments by means of expert knowledge, ICA, Durban, pp. 1604-1615.

Stoter, J., et al., 2009a Methodology for evaluating automated map generalization in commercial software. Computers, Environment and Urban Systems, 33(5), p. 311324.

Tversky, A. (1977). Features of similarity. Psychological Review, 84, pp. 327-352.

Zhang, X., Ai, T., and Stoter, J., 2010. Characterization and detection of building patterns in cartographic data. Joint International Conference on Theory, Data Handling and Modelling in GeoSpatial Information Science (SDH' 2010), Hong Kong, to appear. 